\title{
Research on Visual Design of Creative APP in Big Data
}

\author{
Wang Jiahui \\ Department of Art and Design, Yinxing Hospitality Management College of CUIT, Chengdu, Sichuan, \\ 611743, China \\ email: jiahui1985@163.com
}

Keywords: Big Data Background, Creative App, Visual Design

\begin{abstract}
The rapid development of internet technology under the background of big data, considering the phenomenon of people's needs with a certain differentiation, derived a variety of app software, and the mobile terminal app active users are constantly increasing, further promote the development of app software with its own characteristics. With the emergence of various kinds of creative app and the continuous development of science and technology, but also greatly changed the mass visual aesthetic, with the social trend of the changing mass visual aesthetic, can be said to add difficulty to the development of mobile app under the background of big data, but it is worth noting that in the process of researchers developing app, the core of the mass visual aesthetic harmony is not changing this feature can only grasp the development of app to meet the needs of the public, so there will be colorful Internet products gradually appear. In the development and design of these products, there are often many cross-cutting phenomena, tend to big data and psychological and visual design analysis and inquiry, the purpose of which is to promote the continuous improvement of product performance and user experience.
\end{abstract}

\section{Focus on Holistic Visual Design}

With the increasing number of mobile users, mobile app has gained the opportunity of rapid development, some large websites have transformed into mobile app to expand development, effectively ensure the public convenience needs to meet. The emergence of video app, new media app, friends and games, such as the emergence of various app, greatly enriched the daily life of the public, and the colorful and colorful app interface settings, but also make people's visual aesthetic needs to be fully satisfied. Under the background of the wide application of big data technology and the implementation of related strategies in our country, the research of mobile creative app development based on big data has been strengthened, and when the relevant personnel understand the importance of vision, the research focuses more on the visual research of creative app, which ensures that the public can use app efficiently through the guidance of creative app visual factors, so we should base on the concept of visual design in the research process, put the promotion of mobile app user visual experience on the main goal, strengthen the strength from the aspect of digitization, ensure that the visual design value of creative app under the background of big data can be brought into full play.

Integrity is the first important factor a website should have. When a website has a complicated and confusing clue, the overall control is particularly critical. The color and the structure of the website, such as style and copywriting, are the full embodiment of the design itself and the needs of the users. Under the background of analyzing and considering the needs of users and their psychology, it is necessary to rely on big data to explore the actual needs of users through the full play of the functions of big data collection, analysis and collation, and then consider the integrity of visual design as the basis to ensure that users are guided by visual factors to explore all the clues in the website [1].

for example: take colorful integrity as an example. The color integrity is not only reflected in the basic graphic image of an app, but also the design of some characters should be based on the rules of color integrity elements to ensure that the color of the text and the basic graphic image is 
controlled within the standard color range. In order to ensure the visual unity and integrity of app in the process of designing app, generally speaking, the color of long composition characters in gray and black, and can also be reasonably selected under the background of running other brand standard color and derivative color principles. In general, in the process of designing text color, we should ensure that the brand image is conveyed as much as possible, and provide help for users to find the required information and content quickly, so as to ensure that users can experience better when using app. If you can refer to taobao app and mango tv's app design form, the color can be said to be basically red-orange gradual change form, while the overall form of its app is basically orange-based, the significant advantage of this design is to use color to guide the user's vision, not only to make the user to identify the brand effectively, but also to have a strong psychological role, and these brand design cases are very successful, but also to let the user remember and often use these two app, so in the development of creative app visual design can start with color from this integrity.

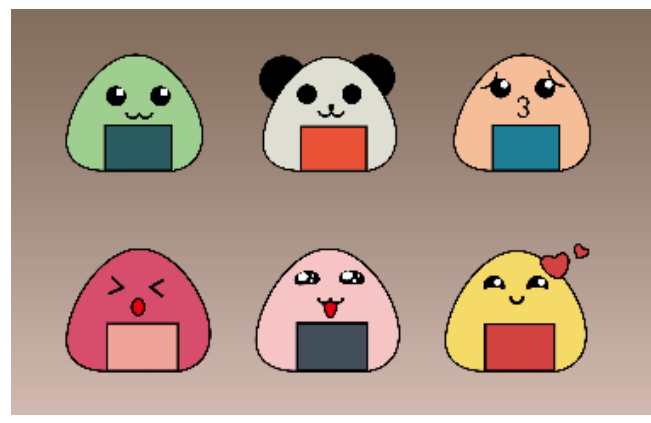

Figure 1 Color integrity

\section{Pays Attention to the Visual Guidance Function of APP}

A mobile app with complicated data and rich content, on the basis of ensuring the overall visual effect, should pay attention to the full play of the role of app guiding users, avoid the user's use of app process, by some irregular information and content caused adverse effects, so that users have the will and desire to use it for a long time. In the process of designing the creative app, to ensure that the user's visual needs can be fully met and thus achieve the guiding purpose, we can divide the block into the starting point, and the app, which has a lot of information content, such as Taobao, JingDong, Su Ning, Amazon and so on, will use the block division to guide the users. [2].

For example: in the creative app development and design process, we can first use the big data technology data tracking and analysis and other functions, mining some of the more popular plate division form. For example, taobao app on the tmall, tmall international and other sections of the division is very popular with users, through these sections can guide users to quickly search for their needs. Therefore, in the creative app design process, you can refer to this plate division form, as far as possible to achieve the clarity of each function of app. On the basis of the clear division of large plates, the rational planning of each sub-plate within it, as far as possible to achieve a clear effect at a glance, can achieve the purpose of guiding users to the sub-plate and each sub-plate to explore. For example, under the background of the rapid development of new media, the rapid development of some new media app has attracted the attention of users, such as quiver app, fast hand app and so on. Taking the app as an example, when we open the app page, we show five interfaces directly at the bottom, and the attention and recommendation of the two sections are subdivided in the functional area of the home page. And the diversity of video content played in the recommended section is also extremely rich and attractive to user research, it is this diversity and do not know what the content of the next video gives users a feeling of irreducible, and it is the characteristics of shaking sound that play a great role in guiding users to improve the user's app usage while meeting the visual needs of user guidance. 


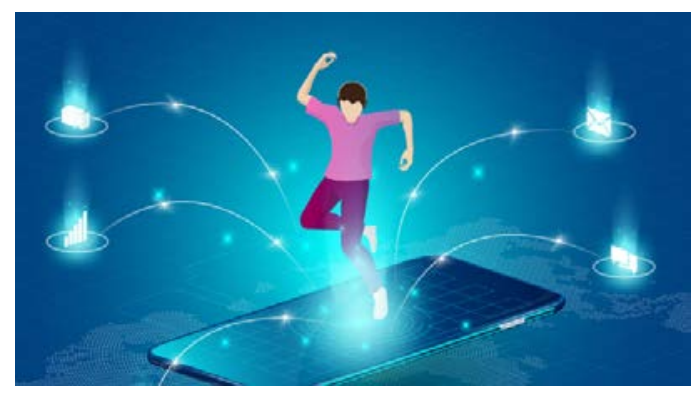

Figure 2 APP visual boot user

\section{Pays Attention to APP user Experience Effect}

In general, most of the mobile app visual design is based on the user characteristics at the present stage. Under the background of grasping the user characteristics as a whole, the factors affecting the user's use of app are analyzed on the basis of fully considering the user's needs, psychological characteristics and emotional experience [3]. From the above two cases, we can also find that in the design of the overall framework and brand image are based on the analysis of user data, and then reasonable planning of app. Such as plate division this design is based on the consideration of road users on the basis of network habits planning division; at the same time, the sub-plate division is also based on user usage and the degree of love and other factors. In the background of big data era, there are many data that can be used to analyze in the process of designing app, so it is not necessary to take the form of single and random sampling as the main way.

Based on the application of big data technology, the more objective and accurate conclusions can also be obtained by big data analysis, and some difficult-to-discovery detail factors can also be obtained by using big data technology, such as the usage habits of some users who may have influence on users and geographical and policy, and economic environment, through the analysis of these more refined and more data information, it can promote the researchers to improve the accuracy of the forecast trend of future development to ensure that the app design meets the visual experience of most users as much as possible.

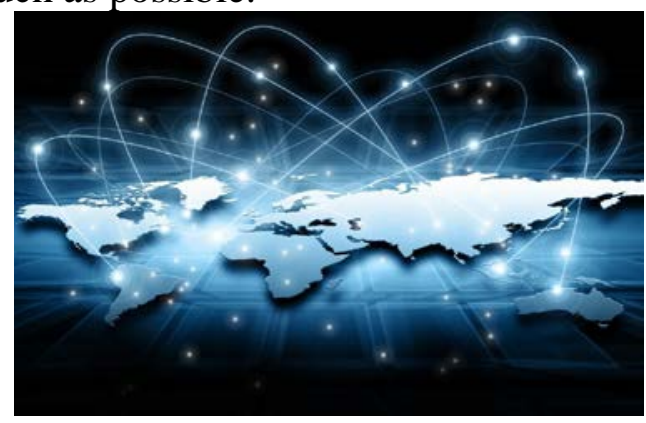

Figure 3 Full use of big data technology

To ensure that the user experience can be gradually improved, user participation is a key measure. Therefore, in the process of developing creative app, we should pay attention to this feature, from the previous user unilateral receiver to two-way interaction as the starting point, and then enhance user participation. With the help of big data technology analysis of this data value has been shown in some dating app and new media app. In general, an app publicity and marketing strategy will be directly affected by the slight changes in user usage habits, so to truly achieve the form of customized app development for users, we should fully and comprehensively consider the needs of users with the help of big data.

\section{Conclusion}

In the process of research on the visual design of the creative app under the background of big data, we can also make research and development from the aspects of interest and function, fully 
utilize the big data technology, fully tap the user's needs and hobbies from the massive information, ensure that the creative app can effectively attract and guide the users from the aspect of visual design, and then provide more data support for the research and development of the creative app, promote the wide application and continuous development of the big data technology, and give full play to the function of the big data technology, With the help of creative app to meet people's visual needs research and development to a great extent enrich people's daily life.

\section{References}

[1] Tan Yuying. A study on the visual design of app interface in Mogao Grottoes Digital Museum. Digital Design (I), no. 11, pp. 25-26, 2019.

[2] Zhu Wei. Analysis of affective visual expression in mobile phone reading class app. Industry and Technology Forum, vol. 18, no. 23, pp. 43-44, 2019.

[3] Chen Kun. Research and application of visual design branding in app interface design. Pop Color, no. 8, pp. 143-144, 2019. 